\title{
SUPPORTING INRORMATION
}

\section{Alkyl spacer length and protonation induced changes in crystalline psychoactive arylpiperazine derivatives: Single-Crystal X-ray, Solid-State NMR, and Computational Studies}

\author{
Edyta Pindelska* ${ }^{a}$, Izabela D. Madura, ${ }^{b}$ Lukasz Szeleszczuk, ${ }^{c}$ Anna Żeszko, ${ }^{a}$ Jolanta \\ Jaśkowska, ${ }^{d}$ Paulina H. Marek, ${ }^{b}$ Waclaw Kolodziejski ${ }^{a}$
}

${ }^{\text {a }}$ Faculty of Pharmacy, Medical University of Warsaw, Department of Inorganic and Analytical Chemistry, Banacha 1, 02-093 Warsaw, Poland.

${ }^{\mathrm{b}}$ Faculty of Chemistry, Warsaw University of Technology, Noakowskiego 3, 00-664 Warsaw, Poland.

${ }^{c}$ Faculty of Pharmacy, Medical University of Warsaw, Department of Physical Chemistry, Banacha 1, 02-093 Warsaw, Poland.

${ }^{\mathrm{d}}$ Cracow University of Technology, Institute of Organic Chemistry and Technology, 24 Warszawska Street, 231-155 Cracow, Poland.

\section{Contents:}

page 2: ${ }^{1} \mathrm{H}$ and ${ }^{13} \mathrm{C}$ NMR in solution

page 4: Table S1. ${ }^{13} \mathrm{C}$ NMR chemical shifts in $\mathrm{CDCl}_{3}$ solution (ppm from TMS) for $\mathbf{L 4 - L 6}$ and L4HCl-L6HCl.

page 6: Table S2. ${ }^{13}$ C CP/MAS NMR chemical shifts (ppm from TMS) for L4-L6 and L4HCl-L6HCl.

page 9: Figure S1. The ${ }^{15} \mathrm{~N}$ CP/MAS NMR spectra of L4-L6 and L4HCl-L6HCl acquired with $5 \mathrm{~ms}$ contact time.

page 10: Table S3. Selected geometrical parameters.

page 13: Figure S2. Molecular fragments used for the calculation of dipole moments: a head (a), a tail (b) and a protonated tail (c).

page 13: Table S4. Values of the ${ }^{13} \mathrm{C}$ and ${ }^{15} \mathrm{~N}$ isotropic (סiso) and calculated chemical shifts anisotropy (CSA) for L4-L6 and L4HCl-L6HCl. 


\section{${ }^{1} \mathrm{H}$ and ${ }^{13} \mathrm{C}$ NMR}

${ }^{1} \mathrm{H}$ and ${ }^{13} \mathrm{C}$ NMR spectra were recorded on a Varian Unity Plus $300 \mathrm{MHz}$ spectrometer in $\mathrm{CDCl}_{3}$ solution with TMS as an internal standard. The spectra data of new compounds refer to their free bases and their hydrochlorides. Chemical shifts were expressed in $\delta$ (ppm) and the coupling constants $\mathrm{J}$ in hertz $(\mathrm{Hz})$. The following abbreviations are used to describe splitting peak patterns when appropriate: s (singlet), $\mathrm{d}$ (doublet), $\mathrm{t}$ (triplet), m (multiplet). Two-dimensional NMR HSQC, COSY, HMBC and NOESY experiments were performed in $\mathrm{CDCl}_{3}$ at $298 \mathrm{~K}$.

Compound L4: ${ }^{1} \mathrm{H}$ NMR $\left(\mathrm{CDCl}_{3}\right) \delta \mathrm{H}: 7.89$ - 7.79 (m, 2H, CH-15, CH-18), 7.75 - 7.67 (m, 2H, CH-16, CH-17), 7.14 (dd, J=7.1, 3.8 Hz, 2H, CH-3, CH-4), $6.99-6.90$ (m, 1H, CH-5), $3.73(\mathrm{t}, \mathrm{J}=7.0 \mathrm{~Hz}, 2 \mathrm{H}, \mathrm{CH} 2-19), 3.06$ (s, 4H, $\left.\mathrm{CH}_{2}-7, \mathrm{CH}_{2}-9\right), 2.64$ (s, 4H, $\left.\mathrm{CH}_{2}-8, \mathrm{CH}_{2}-10\right)$, $2.54-2.40\left(\mathrm{~m}, 2 \mathrm{H}, \mathrm{CH}_{2}-20\right), 1.75\left(\mathrm{dt}, \mathrm{J}=14.4,7.1 \mathrm{~Hz}, 2 \mathrm{H}, \mathrm{CH}_{2}-22\right), 1.66-1.52(\mathrm{~m}, 2 \mathrm{H}$, $\left.\mathrm{CH}_{2}-21\right)$.

Compound L4HCl: ${ }^{1} \mathrm{H}$ NMR $\left(\mathrm{CDCl}_{3}\right) \delta \mathrm{H}: 12.96\left(\mathrm{~s}, 1 \mathrm{H}, \mathrm{NH}^{+}\right), 7.85(\mathrm{dt}, \mathrm{J}=7.1,3.6 \mathrm{~Hz}, 2 \mathrm{H}$, CH-15, CH-18), 7.79 - 7.70 (m, 2H, CH-16, CH-17), 7.30 - 7.14 (m, 2H, CH-3, CH-4), 7.03 (d, J = 7.8 Hz, 1H, CH-5), $3.81-3.53$ (m, 6H, CH-8, CH-10, CH-7, CH-9, CH2-19), 3.38 (d, $\mathrm{J}=12.5 \mathrm{~Hz}, 2 \mathrm{H}, \mathrm{CH}-7, \mathrm{CH}-9), 3.23-3.01\left(\mathrm{~m}, 4 \mathrm{H}, \mathrm{CH}_{2}-20, \mathrm{CH}-8, \mathrm{CH}-10\right), 2.01-1.95$ (m, 2H, $\left.\mathrm{CH}_{2}-21\right), 1.90-1.71\left(\mathrm{~m}, 2 \mathrm{H}, \mathrm{CH}_{2}-22\right)$.

Compound L5: ${ }^{1} \mathrm{H}$ NMR $\left(\mathrm{CDCl}_{3}\right) \delta_{\mathrm{H}}: 7.89-7.78$ (m, 2H, CH-15, CH-18), $7.75-7.67$ (m, 2H, CH-16, CH-17), $7.18-7.08$ (m, 2H, CH-3, CH-4), $7.00-6.90$ (m, 1H, CH-5), $3.75-$ $3.64\left(\mathrm{~m}, 2 \mathrm{H}, \mathrm{CH}_{2}-19\right), 3.04$ (d, J=14.1 Hz, 4H, $\left.\mathrm{CH}_{2}-7, \mathrm{CH}_{2}-9\right), 2.63$ (s, 4H, $\left.\mathrm{CH}_{2}-8, \mathrm{CH}_{2}-10\right)$, 
$2.48-2.35\left(\mathrm{~m}, 2 \mathrm{H}, \mathrm{CH}_{2}-20\right), 1.72\left(\mathrm{dt}, J=15.0,7.5 \mathrm{~Hz}, 2 \mathrm{H}, \mathrm{CH}_{2}-21\right), 1.59(\mathrm{dt}, J=15.2,7.5$ $\left.\mathrm{Hz}, 2 \mathrm{H}, \mathrm{CH}_{2}-22\right), 1.39$ (dt, $J=9.4,6.8 \mathrm{~Hz}, 2 \mathrm{H}, \mathrm{CH}_{2}-23$ ).

Compound L5HCl: ${ }^{1} \mathrm{H}$ NMR $\left(\mathrm{CDCl}_{3}\right) \delta_{\mathrm{H}}: 12.92\left(\mathrm{~s}, 1 \mathrm{H}, \mathrm{NH}^{+}\right), 7.86(\mathrm{dt}, J=7.1,3.5 \mathrm{~Hz}, 2 \mathrm{H}$, CH-15, CH-18), 7.79 - 7.70 (m, 2H, CH-16, CH-17), 7.32 - 7.15 (m, 2H, CH-3, CH-4), 3.82 - 3.56 (m, 6H, CH-7, CH-9, CH-10, $\left.\mathrm{CH}_{2}-19\right), 3.39$ (d, J = 12.7 Hz, 2H, CH-7, CH-9), 3.18 2.94 (m, 4H, CH-8, CH-10, $\left.\mathrm{CH}_{2}-20\right), 2.06$ (s, 2H, $\left.\mathrm{CH}_{2}-23\right), 1.86-1.59$ (m, 2H, $\left.\mathrm{CH}_{2}-21\right), 1.52$ $-1.32\left(\mathrm{~m}, 2 \mathrm{H}, \mathrm{CH}_{2}-22\right)$.

Compound L6: ${ }^{1} \mathrm{H}$ NMR $\left(\mathrm{CDCl}_{3}\right) \delta_{\mathrm{H}}: 7.84(\mathrm{dt}, J=7.4,3.7 \mathrm{~Hz}, 2 \mathrm{H}, \mathrm{CH}-15, \mathrm{CH}-18), 7.75-$ 7.67 (m, 2H, CH-16, CH-17), 7.18 - 7.09 (m, 2H, CH-3, CH-4), $7.00-6.90$ (m, 1H, CH-5), $3.68\left(\mathrm{dd}, J=13.8,6.5 \mathrm{~Hz}, 2 \mathrm{H}, \mathrm{CH}_{2}-19\right), 3.08\left(\mathrm{~s}, 4 \mathrm{H}, \mathrm{CH}_{2}-7, \mathrm{CH}_{2}-9\right), 2.63\left(\mathrm{~s}, 4 \mathrm{H}, \mathrm{CH}_{2}-8\right.$, $\left.\mathrm{CH}_{2}-10\right), 2.42$ (s, 2H, $\left.\mathrm{CH}_{2}-20\right), 1.69$ (d, J = 7.0 Hz, 2H, $\left.\mathrm{CH}_{2}-21\right), 1.54$ (s, 2H, $\left.\mathrm{CH}_{2}-23\right), 1.39$ (d, $\left.J=6.9 \mathrm{~Hz}, 4 \mathrm{H}, \mathrm{CH}_{2}-22, \mathrm{CH}_{2}-24\right)$.

Compound L6HCl: ${ }^{1} \mathrm{H}$ NMR $\left(\mathrm{CDCl}_{3}\right) \delta_{\mathrm{H}}: 12.86\left(\mathrm{~s}, 1 \mathrm{H}, \mathrm{NH}^{+}\right), 7.86(\mathrm{dt}, J=8.5,4.3 \mathrm{~Hz}, 2 \mathrm{H}$, CH-15, CH-18), 7.80 - 7.66 (m, 2H, CH-16, CH-17), 7.30 - 7.13 (m, 2H, CH-3, CH-4), 7.05 (d, J = 7.8 Hz, 1H, CH-5), $3.79-3.56$ (m, 6H, CH $2-19, \mathrm{CH}-7, \mathrm{CH}-8, \mathrm{CH}-9, \mathrm{CH}-10), 3.38$ (d, $J=12.6 \mathrm{~Hz}, 2 \mathrm{H}, \mathrm{CH}-7, \mathrm{CH}-10), 3.07$ (dd, $\left.J=23.0,10.9 \mathrm{~Hz}, 5 \mathrm{H}, \mathrm{CH}_{2}-20, \mathrm{CH}-8, \mathrm{CH}-9\right), 1.99$ (s, 2H, $\left.\mathrm{CH}_{2}-22\right), 1.79-1.61$ (m, 2H, $\left.\mathrm{CH}_{2}-21\right), 1.45$ (s, 4H, $\left.\mathrm{CH}_{2}-23, \mathrm{CH}_{2}-24\right)$. 
Table S1. ${ }^{13} \mathrm{C}$ NMR chemical shifts in $\mathrm{CDCl}_{3}$ solution (ppm from TMS) for L4-L6 and

L4HCl-L6HCl.

\begin{tabular}{|c|c|c|c|c|c|c|c|}
\hline Group & Assignment & $\begin{array}{c}\text { L4 } \\
\delta[\mathrm{ppm}]\end{array}$ & $\begin{array}{l}\mathrm{LAHCl} \\
\delta[\mathrm{ppm}]\end{array}$ & $\begin{array}{c}\text { L5 } \\
\delta[\mathrm{ppm}]\end{array}$ & $\begin{array}{l}\text { L5HCl } \\
\delta[\mathrm{ppm}]\end{array}$ & $\begin{array}{c}\text { L6 } \\
\delta[\mathrm{ppm}]\end{array}$ & $\begin{array}{l}\text { L6HCl } \\
\delta[\mathrm{ppm}]\end{array}$ \\
\hline $\mathrm{C}$ & 11 & 168.19 & 168.60 & 168.93 & 168.64 & 168.47 & 168.65 \\
\hline $\mathrm{C}$ & 12 & 168.19 & 168.60 & 168.93 & 168.64 & 168.47 & 168.65 \\
\hline $\mathrm{C}$ & 2 & 151.47 & 149.16 & 151.78 & 149.16 & 154.85 & 149.15 \\
\hline $\mathrm{C}$ & 6 & 151.47 & 149.16 & 151.78 & 149.16 & 150.45 & 149.15 \\
\hline $\mathrm{CH}$ & 16 & 134.13 & 134.39 & 134.36 & 134.25 & 133.88 & 134.19 \\
\hline $\mathrm{CH}$ & 17 & 134.13 & 134.39 & 134.36 & 134.25 & 133.88 & 134.19 \\
\hline $\mathrm{C}$ & 1 & 134.22 & 132.12 & 134.48 & 134.25 & 134.02 & 134.44 \\
\hline $\mathrm{C}$ & 14 & 132.35 & 132.12 & 132.64 & 132.21 & 132.18 & 132.29 \\
\hline $\mathrm{C}$ & 13 & 127.72 & 127.83 & 127.98 & 127.81 & 132.18 & 127.42 \\
\hline $\mathrm{CH}$ & 4 & 127.65 & 128.08 & 127.90 & 126.39 & 127.45 & 126.42 \\
\hline $\mathrm{CH}$ & 3 & 124.77 & 126.39 & 124.99 & 128.09 & 124.60 & 128.10 \\
\hline $\mathrm{CH}$ & 18 & 123.41 & 123.62 & 123.65 & 123.49 & 123.18 & 123.46 \\
\hline $\mathrm{CH}$ & 15 & 123.41 & 123.62 & 123.65 & 123.49 & 123.18 & 123.37 \\
\hline $\mathrm{CH}$ & 5 & 118.82 & 119.52 & 119.08 & 119.53 & 118.65 & 119.57 \\
\hline $\mathrm{CH}_{2}$ & 20 & 58.16 & 56.92 & 58.84 & 57.58 & 58.52 & 57.73 \\
\hline $\mathrm{CH}_{2}$ & 10 & 53.47 & 52.47 & 53.77 & 52.42 & 53.27 & 48.17 \\
\hline $\mathrm{CH}_{2}$ & 8 & 53.47 & 52.47 & 53.77 & 52.42 & 53.27 & 52.43 \\
\hline $\mathrm{CH}_{2}$ & 7 & 51.43 & 48.16 & 51.73 & 48.16 & 51.15 & 48.17 \\
\hline
\end{tabular}




\begin{tabular}{|c|c|c|c|c|c|c|c|}
\hline $\mathrm{CH}_{2}$ & 9 & 51.43 & 48.16 & 51.73 & 48.16 & 51.15 & 52.43 \\
\hline $\mathrm{CH}_{2}$ & 19 & 38.04 & 36.65 & 38.39 & 37.29 & 37.95 & $\begin{array}{l}37.69 \\
38.09\end{array}$ \\
\hline $\mathrm{CH}_{2}$ & 21 & 26.80 & 26.13 & 28.95 & 28.05 & 28.53 & $\begin{array}{l}28.41 \\
28.65\end{array}$ \\
\hline $\mathrm{CH}_{2}$ & 22 & 24.33 & 20.72 & 26.82 & 22.99 & 26.42 & 23.56 \\
\hline $\mathrm{CH}_{2}$ & 23 & & & 25.27 & 24.08 & 27.09 & $\begin{array}{l}26.47 \\
26.62\end{array}$ \\
\hline $\mathrm{CH}_{2}$ & 24 & & & & & 26.77 & 26.28 \\
\hline
\end{tabular}


Table S2. ${ }^{13} \mathrm{C}$ and ${ }^{15} \mathrm{~N}$ CP/MAS NMR chemical shifts (ppm from TMS) for L4-L6 and

L4HCl-L6HCl. \#

\begin{tabular}{|c|c|c|c|c|c|c|c|}
\hline \multirow[t]{2}{*}{ Atom } & & L4 & L4HCl & L5 & L5HCl & L6 & L6HCI \\
\hline & & $\delta[\mathrm{ppm}]$ & $\delta[\mathrm{ppm}]$ & $\delta[\mathrm{ppm}]$ & $\delta[\mathrm{ppm}]$ & $\delta[\mathrm{ppm}]$ & $\delta[\mathrm{ppm}]$ \\
\hline \multirow[t]{3}{*}{$1-\mathrm{C}$} & $\exp$ & 124.7 & 131.2 & 131.9 & 131.0 & 131.9 & 126.7 \\
\hline & cal & 127.3 & 132.1 & 132.7 & 131.8 & 129.6 & 126.6 \\
\hline & & $(-2.6)$ & $(-0.9)$ & $(-0.8)$ & $(-0.8)$ & (2.3) & $(0.1)$ \\
\hline \multirow[t]{3}{*}{$2-\mathrm{C}$} & $\exp$ & 134.4 & 134.7 & 132.7 & 135.6 & 134.7 & 135.4 \\
\hline & cal & 138.6 & 138.2 & 136.6 & 137.6 & 139.6 & 135.4 \\
\hline & & $(-4.2)$ & $(-3.5)$ & $(-3.9)$ & $(-2.0)$ & $(-4.5)$ & $(0.0)$ \\
\hline \multirow[t]{3}{*}{$3-\mathrm{CH}$} & $\exp$ & 124.7 & 122.9 & 125.1 & 124.6 & 125.8 & 126.3 \\
\hline & cal & 124.9 & 124.7 & 124.9 & 125.2 & 126.0 & 124.8 \\
\hline & & $(-0.2)$ & $(-1.8)$ & $(0.2)$ & $(-0.6)$ & $(-0.2)$ & (1.5) \\
\hline \multirow[t]{3}{*}{$4-\mathrm{CH}$} & $\exp$ & 128.6 & 128.4 & 126.4 & 128.4 & 127.9 & 130.7 \\
\hline & cal & 128.7 & 130.0 & 126.0 & 129.2 & 128.5 & 131.1 \\
\hline & & $(-0.1)$ & $(-1.6)$ & $(0.4)$ & $(-0.8)$ & $(-0.6)$ & $(-0.4)$ \\
\hline \multirow[t]{3}{*}{$5-\mathrm{CH}$} & $\exp$ & 119.7 & 121.4 & 121.7 & 122.5 & 118.7 & 120.0 \\
\hline & cal & 119.9 & 123.0 & 122.5 & 123.4 & 118.8 & 120.4 \\
\hline & & $(-0.2)$ & $(-1.6)$ & $(-0.8)$ & $(-0.9)$ & $(-0.1)$ & $(-0.4)$ \\
\hline \multirow[t]{3}{*}{$6-\mathrm{C}$} & $\exp$ & 150.8 & 150.7 & 151.5 & 150.6 & 151.6 & 148.4 \\
\hline & cal & 151.7 & 152.2 & 152.7 & 152.0 & 152.4 & 149.2 \\
\hline & & $(-0.9)$ & $(-1.5)$ & $(-1.2)$ & $(-1.4)$ & $(-0.8)$ & $(-0.8)$ \\
\hline \multirow[t]{3}{*}{ 7- $\mathrm{CH}_{2}$} & $\exp$ & 45.2 & 47.0 & 50.9 & 47.9 & 51.4 & 47.7 \\
\hline & cal & 46.6 & 45.2 & 48.9 & 46.4 & 49.0 & 44.2 \\
\hline & & $(-1.4)$ & (1.8) & (2.0) & (1.5) & (2.4) & (3.5) \\
\hline \multirow[t]{3}{*}{$8-\mathrm{CH}_{2}$} & $\exp$ & 51.5 & 51.8 & 54.9 & 51.6 & 54.2 & 49.9 \\
\hline & cal & 48.1 & 50.6 & 53.3 & 49.9 & 51.9 & 46.6 \\
\hline & & (3.4) & (1.2) & (1.6) & (1.7) & (2.3) & (3.3) \\
\hline 9- $\mathrm{CH}_{2}$ & $\mathrm{ex}$ & 52.1 & 51.8 & 52.8 & 51.6 & 54.2 & 47.7 \\
\hline
\end{tabular}




\begin{tabular}{|c|c|c|c|c|c|c|}
\hline cal & $\begin{array}{l}49.9 \\
(2.2)\end{array}$ & $\begin{array}{l}48.9 \\
(2.9)\end{array}$ & $\begin{array}{l}49.6 \\
(3.2)\end{array}$ & $\begin{array}{l}48.9 \\
(2.7)\end{array}$ & $\begin{array}{l}51.3 \\
(2.9)\end{array}$ & $\begin{array}{l}43.8 \\
(3.9)\end{array}$ \\
\hline \multirow{3}{*}{$\begin{array}{r}10-\mathrm{CH}_{2} \exp \\
\text { cal }\end{array}$} & 52.9 & 51.8 & 52.8 & 52.4 & 52.2 & 56.5 \\
\hline & 55.2 & 50.4 & 49.9 & 50.8 & 49.9 & 53.9 \\
\hline & $(-2.3)$ & (1.4) & (2.9) & (1.6) & $(2.3)$ & (2.6) \\
\hline \multirow[t]{3}{*}{ 11-C } & 168.7 & 168.4 & 166.6 & 168.7 & 167.5 & 168.7 \\
\hline & 173.0 & 174.1 & 171.5 & 173.4 & 171.7 & 173.9 \\
\hline & $(-4.3)$ & $(-5.7)$ & $(-4.5)$ & $(-4.7)$ & $(-4.2)$ & $(-5.2)$ \\
\hline \multirow[t]{3}{*}{$12-\mathrm{C}$} & 168.7 & 167.0 & 166.6 & 167.1 & 167.5 & 167.6 \\
\hline & 173.7 & 172.3 & 172.3 & 171.8 & 172.4 & 172.9 \\
\hline & $(-5.0)$ & $(-5.3)$ & $(-5.7)$ & $(-4.7)$ & $(-4.9)$ & $(-5.3)$ \\
\hline \multirow{3}{*}{ 13-C } & 133.3 & 132.7 & 131.9 & 131.0 & 131.9 & 132.6 \\
\hline & 133.8 & 133.8 & 132.3 & 131.1 & 132.0 & 133.4 \\
\hline & $(-0.5)$ & $(-1.1)$ & $(-0.4)$ & $(-0.1)$ & $(-0.1)$ & $(-0.8)$ \\
\hline \multirow[t]{3}{*}{$14-C$} & 132.1 & 131.2 & 132.7 & 133.5 & 131.9 & 132.6 \\
\hline & 132.0 & 132.0 & 133.1 & 133.0 & 132.1 & 133.4 \\
\hline & $(0.1)$ & $(-0.8)$ & $(-0.4)$ & $(0.5)$ & $(-0.2)$ & $(-0.8)$ \\
\hline \multirow[t]{3}{*}{$15-\mathrm{CH}$} & 124.7 & 122.9 & 119.6 & 122.5 & 122.7 & 126.3 \\
\hline & 127.1 & 124.7 & 120.1 & 123.4 & 124.1 & 128.1 \\
\hline & $(-2.4)$ & $(-1.8)$ & $(-0.5)$ & $(-0.9)$ & $(-1.4)$ & $(-1.8)$ \\
\hline \multirow[t]{3}{*}{$16-\mathrm{CH}$} & 134.4 & 134.7 & 132.7 & 133.5 & 131.9 & 135.4 \\
\hline & 136.8 & 137.9 & 134.9 & 135.2 & 134.2 & 137.6 \\
\hline & $(-2.4)$ & $(-3.2)$ & $(-2.2)$ & $(-1.7)$ & $(-2.3)$ & $(-2.2)$ \\
\hline \multirow[t]{3}{*}{ 17-CH } & 133.3 & 132.7 & 136.3 & 135.6 & 134.7 & 132.6 \\
\hline & 136.0 & 135.5 & 138.9 & 138.3 & 135.2 & 134.7 \\
\hline & $(-2.7)$ & $(-2.8)$ & $(-2.6)$ & $(-2.7)$ & $(-0.5)$ & $(-2.1)$ \\
\hline \multirow[t]{3}{*}{$18-\mathrm{CH} \exp$} & 124.7 & 122.9 & 126.4 & 122.5 & 122.7 & 123.0 \\
\hline & 125.4 & 124.5 & 128.5 & 125.2 & 123.1 & 124.2 \\
\hline & $(-0.7)$ & $(-1.6)$ & $(-2.1)$ & $(-2.7)$ & $(-0.4)$ & $(-1.2)$ \\
\hline $19-\mathrm{CH}_{2} \exp$ & 37.9 & 37.4 & 37.4 & 37.8 & 37.7 & 38.9 \\
\hline
\end{tabular}




\begin{tabular}{|c|c|c|c|c|c|c|}
\hline cal & $\begin{array}{l}36.3 \\
(1.6)\end{array}$ & $\begin{array}{l}35.6 \\
(1.8)\end{array}$ & $\begin{array}{l}35.4 \\
(2.0)\end{array}$ & $\begin{array}{l}35.1 \\
(2.7)\end{array}$ & $\begin{array}{l}35.8 \\
(1.9)\end{array}$ & $\begin{array}{l}37.2 \\
(1.7)\end{array}$ \\
\hline $20-\mathrm{CH}_{2} \exp$ & 58.0 & 56.0 & 60.3 & 56.5 & 59.1 & 57.6 \\
\hline \multirow[t]{2}{*}{ cal } & 57.9 & 55.2 & 59.2 & 55.7 & 58.2 & 56.0 \\
\hline & $(0.1)$ & $(0.8)$ & (1.1) & $(0.8)$ & $(0.9)$ & (1.6) \\
\hline $21-\mathrm{CH}_{2} \exp$ & 27.2 & 25.3 & 31.4 & 28.6 & 28.6 & 30.9 \\
\hline \multirow[t]{2}{*}{ cal } & 25.6 & 22.8 & 29.0 & 26.3 & 26.3 & 29.3 \\
\hline & (1.6) & $(2.5)$ & (2.4) & (2.3) & (2.3) & (1.6) \\
\hline $22-\mathrm{CH}_{2} \exp$ & 24.2 & 20.4 & 28.8 & 25.0 & 31.8 & 25.3 \\
\hline \multirow[t]{2}{*}{ cal } & 21.6 & 16.4 & 26.0 & 20.7 & 29.4 & 21.9 \\
\hline & (2.6) & $(4.0)$ & (2.8) & $(4.3)$ & (2.4) & (3.4) \\
\hline \multicolumn{2}{|l|}{$23-\mathrm{CH}_{2} \exp$} & & 26.2 & 25.0 & 28.6 & 28.1 \\
\hline \multirow[t]{2}{*}{$\mathrm{cal}$} & & & 23.3 & 22.0 & 26.0 & 25.8 \\
\hline & & & (2.9) & (3.0) & (2.6) & (2.3) \\
\hline \multicolumn{2}{|l|}{$24-\mathrm{CH}_{2} \exp$} & & & & 26.2 & 27.2 \\
\hline \multirow[t]{2}{*}{ cal } & & & & & 22.8 & 24.4 \\
\hline & & & & & (3.4) & (2.8) \\
\hline \multirow[t]{3}{*}{$\mathrm{N}-1$} & -314.3 & -322.6 & -320.0 & -321.7 & -312.7 & -315.2 \\
\hline & -306.0 & -313.9 & -313.7 & -313.4 & -305.4 & -307.6 \\
\hline & $(-8.3)$ & $(-8.7)$ & $(-6.3)$ & $(-8.3)$ & $(-7.3)$ & $(-7.6)$ \\
\hline \multirow[t]{3}{*}{$\mathrm{N}-2$} & -332.9 & -326.3 & -330.4 & -325.7 & -331.1 & -324.2 \\
\hline & -331.5 & -317.5 & -328.6 & -318.3 & -329.0 & -318.1 \\
\hline & $(-1.4)$ & $(-8.8)$ & $(-1.8)$ & $(-7.4)$ & $(-2.1)$ & $(-6.1)$ \\
\hline \multirow[t]{3}{*}{$\mathrm{N}-3$} & -213.7 & -213.6 & -214.1 & -214.9 & -214.8 & -215.6 \\
\hline & -196.0 & -195.3 & -197.5 & -197.0 & -197.9 & -198.0 \\
\hline & $(-17.7)$ & $(-18.3)$ & $(-16.6)$ & $(-17.9)$ & $(-16.9)$ & $(-17.6)$ \\
\hline
\end{tabular}

\# Values in brackets: ( $\delta$ experimental $-\delta$ computed); CASTEP computed $\delta$ values have been obtained from all atoms positions optimization (the lattice parameters were fixed to their experimental values). 


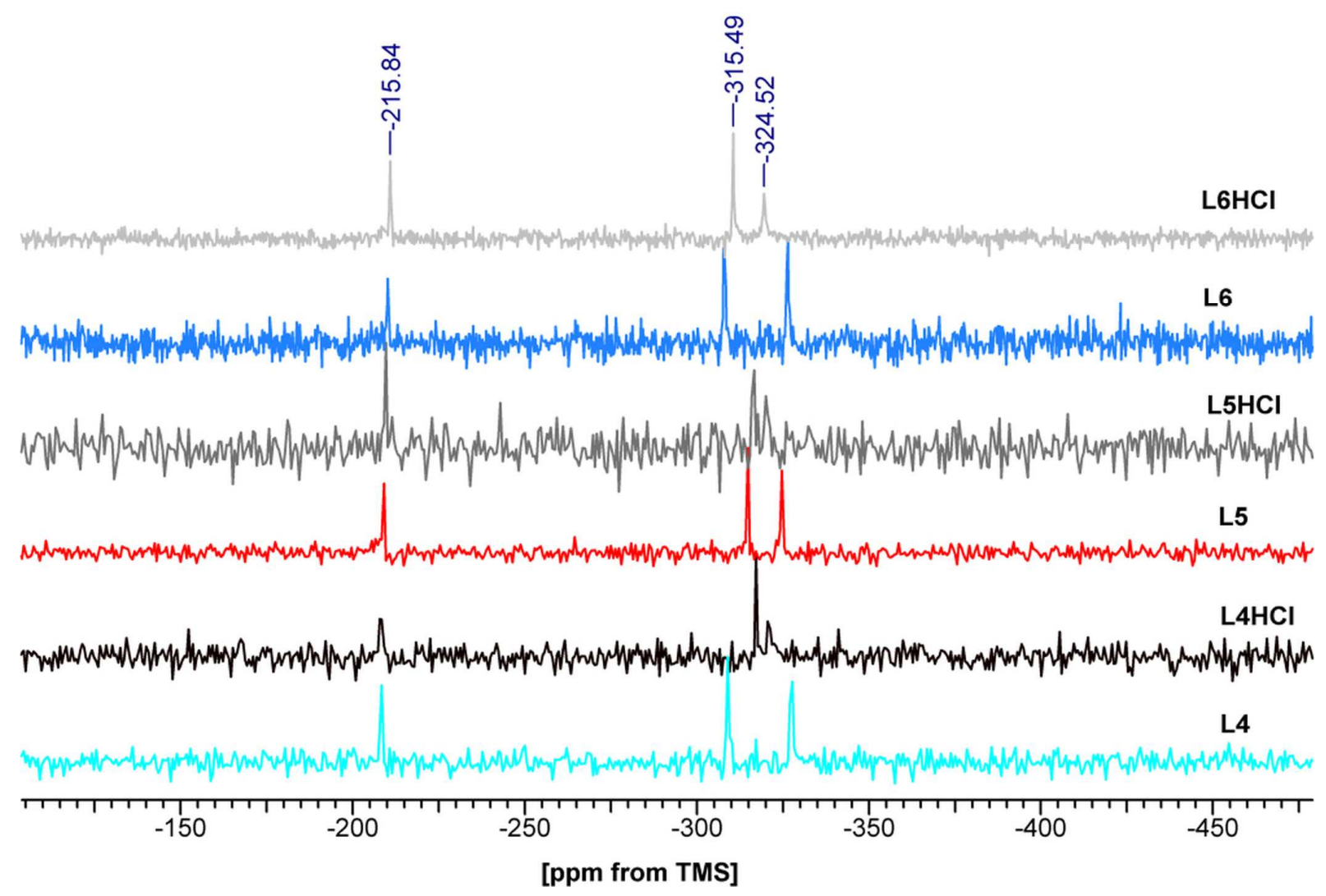

Figure S1. The ${ }^{15} \mathrm{~N}$ CP/MAS NMR spectra of L4-L6 and L4HCl-L6HCl acquired with 5 ms contact time. 
Table S3. Selected geometrical parameters.

\begin{tabular}{|c|c|c|c|c|c|c|}
\hline & L4 & L5 & L6 & L4HCl & L5HCl & L6HCl \\
\hline \multicolumn{7}{|l|}{ BONDS (Å) } \\
\hline C1-Cl1 & $1.732(2)$ & $1.728(2)$ & $1.734(1)$ & $1.727(2)$ & $1.728(4)$ & $1.729(2)$ \\
\hline $\mathrm{C} 2-\mathrm{Cl} 2$ & $1.733(2)$ & $1.736(2)$ & $1.728(2)$ & $1.732(3)$ & $1.735(5)$ & $1.740(2)$ \\
\hline C6-N1 & $1.409(3)$ & $1.418(2)$ & $1.409(2)$ & $1.407(3)$ & $1.413(5)$ & $1.413(2)$ \\
\hline C7-N1 & $1.465(3)$ & $1.469(2)$ & $1.466(2)$ & $1.458(2)$ & $1.468(5)$ & $1.456(2)$ \\
\hline C9-N1 & $1.470(3)$ & $1.456(2)$ & $1.473(2)$ & $1.464(3)$ & $1.463(4)$ & $1.470(2)$ \\
\hline C7-C8 & $1.511(4)$ & $1.511(2)$ & $1.510(2)$ & $1.509(3)$ & $1.503(5)$ & $1.508(2)$ \\
\hline C9-C10 & $1.499(4)$ & $1.507(2)$ & $1.506(2)$ & $1.499(3)$ & $1.506(5)$ & $1.511(2)$ \\
\hline C8-N2 & $1.452(3)$ & $1.461(2)$ & $1.461(2)$ & $1.497(2)$ & $1.495(4)$ & $1.492(2)$ \\
\hline C10-N2 & $1.454(4)$ & $1.461(2)$ & $1.458(2)$ & $1.498(2)$ & $1.492(4)$ & $1.497(2)$ \\
\hline C20-N2 & $1.480(4)$ & $1.462(2)$ & $1.459(2)$ & $1.493(2)$ & $1.497(4)$ & $1.496(2)$ \\
\hline C11-01 & $1.203(3)$ & $1.204(2)$ & $1.198(2)$ & $1.210(3)$ & $1.213(6)$ & $1.210(2)$ \\
\hline $\mathrm{C} 12-\mathrm{O} 2$ & $1.204(4)$ & $1.201(2)$ & $1.198(2)$ & $1.204(3)$ & $1.203(7)$ & $1.201(2)$ \\
\hline C11-N3 & $1.384(4)$ & $1.386(3)$ & $1.386(2)$ & $1.390(3)$ & $1.380(7)$ & $1.387(2)$ \\
\hline C12-N3 & $1.390(4)$ & $1.394(2)$ & $1.386(2)$ & $1.387(3)$ & $1.400(6)$ & $1.399(2)$ \\
\hline C19-N3 & $1.437(4)$ & $1.458(2)$ & $1.457(5)$ & $1.463(3)$ & $1.462(6)$ & $1.462(2)$ \\
\hline \multicolumn{7}{|l|}{ ANGLES $\left({ }^{\circ}\right)$} \\
\hline C1-C6-N1 & $120.2(2)$ & $119.47(14)$ & $120.32(12)$ & $118.6(2)$ & $118.7(3)$ & $120.63(14)$ \\
\hline C5-C6-C1 & $117.1(2)$ & $117.58(15)$ & $117.04(13)$ & $118.0(2)$ & $118.2(3)$ & $117.12(15)$ \\
\hline C5-C6-N1 & $122.6(2)$ & $122.91(15)$ & $122.57(13)$ & $123.4(2)$ & $123.0(3)$ & $122.18(15)$ \\
\hline N1-C7-C8 & $109.3(2)$ & $110.06(14)$ & $110.00(11)$ & $108.0(2)$ & $108.7(3)$ & $109.89(14)$ \\
\hline N2-C8-C7 & $112.1(2)$ & $111.55(13)$ & $111.63(12)$ & $110.56(15)$ & $110.1(3)$ & $111.13(14)$ \\
\hline N1-C9-C10 & $109.6(2)$ & $109.39(13)$ & $109.94(11)$ & $110.55(16)$ & $110.6(3)$ & $109.49(14)$ \\
\hline $\mathrm{N} 2-\mathrm{C} 10-\mathrm{C} 9$ & $111.0(2)$ & $111.27(14)$ & $110.50(12)$ & $111.12(16)$ & $111.0(3)$ & $110.31(13)$ \\
\hline
\end{tabular}




\begin{tabular}{|c|c|c|c|c|c|c|}
\hline C6-N1-C7 & 116.07(19) & $114.43(13)$ & $116.59(11)$ & $117.99(15)$ & $116.8(3)$ & $116.47(13)$ \\
\hline C6-N1-C9 & $115.75(18)$ & $115.31(12)$ & $115.03(11)$ & $115.37(15)$ & $113.7(3)$ & $116.71(13)$ \\
\hline C7-N1-C9 & 108.84(18) & $109.30(13)$ & $108.42(11)$ & 109.21(15) & 109.3(3) & 108.78(13) \\
\hline C8-N2-C10 & $108.8(2)$ & 108.81(12) & $109.22(11)$ & $110.25(14)$ & $109.7(2)$ & $109.48(13)$ \\
\hline C20-N2-C8 & $113.3(2)$ & $111.72(13)$ & $110.44(11)$ & $113.45(15)$ & $113.4(3)$ & $112.68(14)$ \\
\hline C20-N2-C10 & $107.5(2)$ & $111.72(13)$ & $112.63(11)$ & $109.93(14)$ & $110.1(2)$ & 111.38(13) \\
\hline C11-N3-C19 & $123.5(3)$ & $124.30(15)$ & $123.2(3)$ & $124.8(2)$ & $124.3(4)$ & $124.04(15)$ \\
\hline C12-N3-C11 & $112.5(2)$ & $112.19(15)$ & $113.6(4)$ & $111.7(2)$ & $111.9(4)$ & $111.66(13)$ \\
\hline C12-N3-C19 & $123.1(2)$ & $123.43(16)$ & $123.2(4)$ & $123.3(2)$ & $123.7(4)$ & $123.95(14)$ \\
\hline \multicolumn{7}{|l|}{$\begin{array}{c}\text { TORSION } \\
\left.\text { ANGLES ( }{ }^{\circ}\right)\end{array}$} \\
\hline N1-C7-C8-N2 & $58.4(3)$ & $57.65(19)$ & $58.2(2)$ & $61.3(2)$ & $56.2(4)$ & $58.8(2)$ \\
\hline N1-C9-C10-N2 & $-60.6(3)$ & $-60.53(19)$ & $-60.7(2)$ & $-55.9(2)$ & $-61.9(4)$ & $-59.9(2)$ \\
\hline C1-C6-N1-C7 & $-158.2(2)$ & $-80.5(2)$ & $-162.1(1)$ & $-150.6(2)$ & $-80.8(4)$ & $-165.0(2)$ \\
\hline C1-C6-N1-C9 & $72.4(3)$ & $151.5(2)$ & $69.4(2)$ & $77.8(2)$ & $150.4(3)$ & $64.2(2)$ \\
\hline C5-C6-N1-C7 & $19.5(3)$ & $101.8(2)$ & $14.8(2)$ & $26.7(3)$ & $101.6(4)$ & $11.7(2)$ \\
\hline C5-C6-N1-C9 & $-109.9(3)$ & $-26.2(2)$ & $-113.8(2)$ & $-105.0(2)$ & $-27.2(5)$ & $-119.1(2)$ \\
\hline C7-C8-N2-C20 & $-176.6(2)$ & $-177.5(1)$ & $178.8(1)$ & $-178.9(1)$ & $-179.6(3)$ & $-179.4(2)$ \\
\hline C9-C10-N2-C20 & $-179.0(2)$ & $-179.7(1)$ & $-179.1(1)$ & $177.9(2)$ & $-179.4(3)$ & $-179.4(2)$ \\
\hline $\begin{array}{l}\mathrm{C} 8-\mathrm{N} 2-\mathrm{C} 20-\mathrm{C} 22 \\
(\tau)\end{array}$ & $-58.1(4)$ & $-71.7(2)$ & $-170.7(1)$ & $-68.8(2)$ & $-169.7(3)$ & $-71.6(2)$ \\
\hline C10-N2-C20-C22 & $-178.3(3)$ & $167.7(1)$ & $66.9(2)$ & $167.2(2)$ & $67.0(4)$ & $164.9(2)$ \\
\hline Cl1-C1-C6-N1 & $-0.7(3)$ & $2.4(2)$ & $3.0(2)$ & $0.2(3)$ & $0.3(4)$ & $5.0(2)$ \\
\hline \multirow[t]{2}{*}{ 01-C11-N3-C19 } & $-9.0(4)$ & $-4.0(3)$ & $0.6(9)$ & $5.6(4)$ & $5.2(6)$ & $4.1(3)$ \\
\hline & & & $0.9(8)$ & & & \\
\hline \multirow[t]{2}{*}{ O2-C12-N3-C19 } & $9.8(5)$ & $4.7(3)$ & $-2.1(7)$ & $-5.2(3)$ & $-4.0(7)$ & $-6.8(3)$ \\
\hline & & & $2.2(7)$ & & & \\
\hline C21-C19-N3-C12 & $-103.2(4)$ & $79.9(2)$ & $83.0(5)$ & $-91.7(3)$ & $-89.3(7)$ & $73.4(2)$ \\
\hline
\end{tabular}




\begin{tabular}{|c|c|c|c|c|c|c|}
\hline \multirow{3}{*}{ C21-C19-N3-C11 } & \multirow{3}{*}{$88.3(4)$} & \multirow{3}{*}{$-96.6(2)$} & \multicolumn{2}{|l|}{$-85.6(9)$} & \multicolumn{2}{|l|}{$-114.5(9)$} \\
\hline & & & $-101.0(5)$ & $83.1(3)$ & $87.2(7)$ & $-113.9(2)$ \\
\hline & & & \multicolumn{2}{|l|}{$95.9(9)$} & \multicolumn{2}{|l|}{$62.0(9)$} \\
\hline $\begin{array}{l}\text { Puckering } \\
\text { Amplitude (Q/A) }\end{array}$ & $0.588(3)$ & $0.585(2)$ & $0.590(2)$ & $0.593(2)$ & $0.594(4)$ & $0.596(2)$ \\
\hline Puckering $\left(\theta /^{\circ}\right)$ & $2.6(3)$ & $2.2(2)$ & $2.6(1)$ & $6.6(2)$ & $5.1(4)$ & $4.0(2)$ \\
\hline Puckering $\left(\varphi /^{\circ}\right)$ & $329(6)$ & $225(4)$ & $323(3)$ & $32(2)$ & $227(4)$ & $346(3)$ \\
\hline piper-tail $\left({ }^{\circ}\right)$ & $45.9(1)$ & $54.50(9)$ & $42.01(8)$ & $53.5(1)$ & $55.5(2)$ & $36.93(9)$ \\
\hline \multirow[t]{2}{*}{ piper-head $\left({ }^{\circ}\right)$} & \multirow[t]{2}{*}{$62.6(1)$} & \multirow[t]{2}{*}{$49.2(1)$} & $34.9(2)$ & \multirow[t]{2}{*}{$57.9(1)$} & \multirow[t]{2}{*}{$59.4(2)$} & \multirow[t]{2}{*}{$11.6(1)$} \\
\hline & & & $33.4(5)$ & & & \\
\hline \multirow[t]{2}{*}{ head-tail $\left(^{\circ}\right)$} & \multirow[t]{2}{*}{$71.6(1)$} & \multirow[t]{2}{*}{$64.2(1)$} & $74.6(3)$ & \multirow[t]{2}{*}{$5.3(1)$} & \multirow[t]{2}{*}{$27.3(2)$} & \multirow[t]{2}{*}{$47.17(9)$} \\
\hline & & & $75.0(5)$ & & & \\
\hline
\end{tabular}




\section{Dipole moments}

The ground-state dipole moment of "a head", "a tail" and "a protonated tail" was calculated by quantum chemical calculations. All calculations were carried out using the DMol3 program package, implemented in the Materials Studio 6.1. software. ${ }^{1}{ }^{3}$

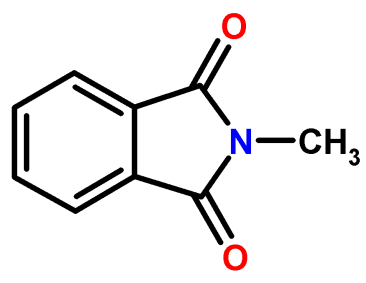

(a)

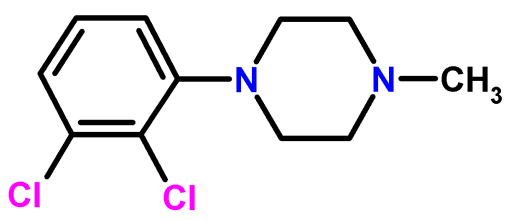

(b)

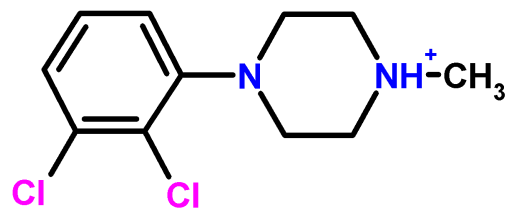

(c)

Figure S2. Molecular fragments used for the calculation of dipole moments: a head (a), a tail (b) and a protonated tail (c).

Table S4. Values of the ${ }^{13} \mathrm{C}$ and ${ }^{15} \mathrm{~N}$ isotropic (סiso) and calculated chemical shifts anisotropy (CSA) for L4-L6 and L4HCl-L6HCl.

\begin{tabular}{|c|c|c|c|c|c|c|}
\hline \multirow{3}{*}{ Atom } & \multicolumn{3}{|c|}{ L4 } & \multicolumn{3}{|c|}{ L4HCl } \\
\hline & $\begin{array}{l}\text { Isotropic } \\
\text { [ppm] }\end{array}$ & $\begin{array}{l}\text { Anisotropy } \\
\text { [ppm] }\end{array}$ & $\begin{array}{c}\text { Asymmetry } \\
\eta\end{array}$ & $\begin{array}{l}\text { Isotropic } \\
\text { [ppm] }\end{array}$ & $\begin{array}{c}\text { Anisotropy } \\
\text { [ppm] }\end{array}$ & $\begin{array}{c}\text { Asymmetry } \\
\eta\end{array}$ \\
\hline & Siso & $\Delta \delta$ & & Eiso & $\Delta \delta$ & \\
\hline $1-\mathrm{C}$ & 42.15 & -107.17 & 0.54 & 37.42 & -110.03 & 0.69 \\
\hline $2-\mathrm{C}$ & 30.88 & -132.93 & 0.61 & 31.28 & -134.3 & 0.61 \\
\hline $3-\mathrm{CH}$ & 44.61 & 150.48 & 0.83 & 44.78 & 146.35 & 0.87 \\
\hline $4-\mathrm{CH}$ & 40.77 & 186.67 & 0.72 & 39.52 & 191.73 & 0.66 \\
\hline $5-\mathrm{CH}$ & 49.55 & 177.12 & 0.6 & 46.47 & 177.59 & 0.61 \\
\hline $6-\mathrm{C}$ & 17.82 & 153.98 & 0.38 & 17.28 & 153.29 & 0.42 \\
\hline 7- $\mathrm{CH}_{2}$ & 122.87 & 28.97 & 0.41 & 124.27 & 38.1 & 0.31 \\
\hline $8-\mathrm{CH}_{2}$ & 121.38 & -36.41 & 0.69 & 118.91 & -30.91 & 0.78 \\
\hline
\end{tabular}

${ }^{1}$ Delley, B. J. Chem. Phys. 1990, 92, 508-517.

${ }^{2}$ Delley, B. J. Phys. Chem. 1996, 100, 6107-6110.

${ }^{3}$ Delley, B. J. Chem. Phys. 2000, 113, 7756-7764. 


\begin{tabular}{|c|c|c|c|c|c|c|}
\hline 9- $\mathrm{CH}_{2}$ & 119.63 & 29.47 & 0.85 & 120.58 & 44.5 & 0.38 \\
\hline $10-\mathrm{CH}_{2}$ & 114.32 & 40.03 & 0.73 & 119.04 & 34.29 & 0.56 \\
\hline $11-\mathrm{C}$ & -3.55 & -125.55 & 0.57 & -4.66 & -127.11 & 0.64 \\
\hline $12-\mathrm{C}$ & -4.18 & -125.69 & 0.61 & -2.82 & -126 & 0.53 \\
\hline $13-\mathrm{C}$ & 35.67 & 170.55 & 0.71 & 35.65 & 169.52 & 0.73 \\
\hline $14-\mathrm{C}$ & 37.5 & 170.57 & 0.71 & 37.46 & 168.01 & 0.68 \\
\hline $15-\mathrm{CH}$ & 42.35 & 199.53 & 0.42 & 44.83 & 194.71 & 0.45 \\
\hline $16-\mathrm{CH}$ & 32.69 & 202.17 & 0.62 & 31.63 & 206.4 & 0.54 \\
\hline $17-\mathrm{CH}$ & 33.52 & 203.73 & 0.59 & 34.02 & 199.67 & 0.66 \\
\hline $18-\mathrm{CH}$ & 44.1 & 198.81 & 0.43 & 44.98 & 196.62 & 0.49 \\
\hline $19-\mathrm{CH}_{2}$ & 133.16 & -57.12 & 0.29 & 133.87 & -63.51 & 0.47 \\
\hline $20-\mathrm{CH}_{2}$ & 111.6 & 65.55 & 0.25 & 114.31 & 67.42 & 0.64 \\
\hline $21-\mathrm{CH}_{2}$ & 143.9 & 29.79 & 0.79 & 146.72 & -37.77 & 0.75 \\
\hline $22-\mathrm{CH}_{2}$ & 147.91 & -20.22 & 0.92 & 153.09 & 22.26 & 0.36 \\
\hline $\mathrm{N}-1$ & 155.6 & -63.88 & 0.47 & 163.49 & -51.79 & 0.21 \\
\hline $\mathrm{N}-2$ & 181.12 & 31.76 & 0.98 & 167.12 & 24.9 & 0.61 \\
\hline \multirow[t]{2}{*}{$\mathrm{N}-3$} & 45.59 & 127.63 & 0.3 & 44.92 & 131.12 & 0.18 \\
\hline & & L5 & & & L5HCl & \\
\hline \multirow[t]{2}{*}{ Atom } & $\begin{array}{l}\text { Isotropic } \\
\text { [ppm] }\end{array}$ & $\begin{array}{c}\text { Anisotropy } \\
\text { [ppm] }\end{array}$ & $\begin{array}{c}\text { Asymmetry } \\
\eta\end{array}$ & $\begin{array}{c}\text { Isotropic } \\
\text { [ppm] }\end{array}$ & $\begin{array}{l}\text { Anisotropy } \\
\text { [ppm] }\end{array}$ & $\begin{array}{c}\text { Asymmetry } \\
\eta\end{array}$ \\
\hline & Siso & $\Delta \delta$ & & Siso & $\Delta \delta$ & \\
\hline 1-C & 36.82 & -108.56 & 0.71 & 37.67 & -110.87 & 0.72 \\
\hline $2-\mathrm{C}$ & 32.93 & -131.22 & 0.56 & 31.9 & -133.98 & 0.62 \\
\hline $3-\mathrm{CH}$ & 44.62 & 150.46 & 0.86 & 44.27 & 196.27 & 0.44 \\
\hline $4-\mathrm{CH}$ & 43.45 & 185.15 & 0.72 & 40.31 & 194.36 & 0.63 \\
\hline $5-\mathrm{CH}$ & 47 & 184.3 & 0.6 & 46.09 & 194.01 & 0.49 \\
\hline $6-\mathrm{C}$ & 16.76 & 154.02 & 0.44 & 17.44 & 154.87 & 0.43 \\
\hline $7-\mathrm{CH}_{2}$ & 120.61 & 29.98 & 0.25 & 123.12 & 35.12 & 0.35 \\
\hline $8-\mathrm{CH}_{2}$ & 116.22 & 42.06 & 0.17 & 119.54 & -30.82 & 0.91 \\
\hline 9- $\mathrm{CH}_{2}$ & 119.84 & 33.11 & 0.79 & 120.59 & 45.1 & 0.4 \\
\hline $10-\mathrm{CH}_{2}$ & 119.58 & -39.99 & 0.74 & 118.72 & 38.1 & 0.48 \\
\hline
\end{tabular}




\begin{tabular}{|c|c|c|c|c|c|c|}
\hline $11-C$ & -2.03 & -128.05 & 0.48 & -3.96 & -126.7 & 0.66 \\
\hline $12-C$ & -2.79 & -129.74 & 0.52 & -2.3 & -126.03 & 0.56 \\
\hline $13-\mathrm{C}$ & 37.18 & 166.62 & 0.75 & 38.41 & 167.49 & 0.7 \\
\hline $14-C$ & 36.35 & 168.54 & 0.73 & 36.48 & 168.18 & 0.73 \\
\hline $15-\mathrm{CH}$ & 49.36 & 188.56 & 0.51 & 46.05 & 180.88 & 0.58 \\
\hline $16-\mathrm{CH}$ & 34.62 & 202.09 & 0.64 & 34.32 & 202.73 & 0.64 \\
\hline $17-\mathrm{CH}$ & 30.61 & 209.72 & 0.56 & 31.23 & 208.38 & 0.53 \\
\hline $18-\mathrm{CH}$ & 41.01 & 205.1 & 0.4 & 44.24 & 149.11 & 0.88 \\
\hline $19-\mathrm{CH}_{2}$ & 134.13 & -53.64 & 0.56 & 134.43 & -63.28 & 0.45 \\
\hline $20-\mathrm{CH}_{2}$ & 110.28 & 67.37 & 0.35 & 113.81 & 59.87 & 0.68 \\
\hline $21-\mathrm{CH}_{2}$ & 140.52 & -35.64 & 0.69 & 143.21 & 33.33 & 0.94 \\
\hline $22-\mathrm{CH}_{2}$ & 143.52 & -28.71 & 0.5 & 148.8 & -27.53 & 0.38 \\
\hline $23-\mathrm{CH}_{2}$ & 146.19 & 19.75 & 0.82 & 147.5 & 17.77 & 0.74 \\
\hline $\mathrm{N}-1$ & 163.28 & -52.93 & 0.44 & 162.95 & -47.71 & 0.3 \\
\hline $\mathrm{N}-2$ & 178.18 & -29.42 & 0.84 & 167.88 & 26.32 & 0.59 \\
\hline \multirow[t]{2}{*}{$\mathrm{N}-3$} & 47.08 & 125.79 & 0.28 & 46.61 & 131.42 & 0.22 \\
\hline & & L6 & & & L6HCl & \\
\hline Atom & $\begin{array}{c}\text { Isotropic } \\
\text { [ppm] } \\
\text { Siso }\end{array}$ & $\begin{array}{l}\text { Anisotropy } \\
{[\mathrm{ppm}] \Delta \delta}\end{array}$ & $\begin{array}{c}\text { Asymmetry } \\
\eta\end{array}$ & $\begin{array}{c}\text { Isotropic } \\
\text { [ppm] } \\
\text { Siso }\end{array}$ & $\begin{array}{c}\text { Anisotropy } \\
{[\mathrm{ppm}] \Delta \delta}\end{array}$ & $\begin{array}{c}\text { Asymmetry } \\
\eta\end{array}$ \\
\hline $1-C$ & 39.87 & -107.36 & 0.62 & 42.83 & -107.14 & 0.72 \\
\hline $2-\mathrm{C}$ & 29.91 & -132.7 & 0.57 & 34.08 & -133.12 & 0.44 \\
\hline $3-\mathrm{CH}$ & 43.52 & 155.87 & 0.76 & 44.68 & 150.14 & 0.8 \\
\hline $4-\mathrm{CH}$ & 40.96 & 181.8 & 0.77 & 38.41 & 191.05 & 0.64 \\
\hline $5-\mathrm{CH}$ & 50.68 & 171.59 & 0.62 & 49.05 & 178.5 & 0.57 \\
\hline 6-C & 17.08 & 153.2 & 0.38 & 20.28 & 154.12 & 0.4 \\
\hline 7- $\mathrm{CH}_{2}$ & 120.53 & 28 & 0.55 & 125.31 & 31.85 & 0.54 \\
\hline $8-\mathrm{CH}_{2}$ & 117.54 & 42.1 & 0.05 & 122.86 & -35.39 & 0.9 \\
\hline 9- $\mathrm{CH}_{2}$ & 118.18 & 31.92 & 0.88 & 125.7 & 36.05 & 0.6 \\
\hline $10-\mathrm{CH}_{2}$ & 119.54 & -37.7 & 0.64 & 115.54 & 39.53 & 0.52 \\
\hline $11-C$ & -2.17 & -127.52 & 0.5 & -4.38 & -123.43 & 0.64 \\
\hline $12-\mathrm{C}$ & -2.89 & -123.14 & 0.58 & -3.41 & -128.96 & 0.46 \\
\hline
\end{tabular}




\begin{tabular}{lllllll}
\hline $13-\mathrm{C}$ & 37.48 & 167.7 & 0.72 & 36.11 & 164.85 & 0.74 \\
\hline $14-\mathrm{C}$ & 37.34 & 168.91 & 0.71 & 36.13 & 167.38 & 0.76 \\
\hline $15-\mathrm{CH}$ & 45.42 & 196.9 & 0.45 & 41.34 & 201.88 & 0.38 \\
\hline $16-\mathrm{CH}$ & 35.31 & 197.77 & 0.66 & 31.88 & 204.2 & 0.61 \\
\hline $17-\mathrm{CH}$ & 34.27 & 199.54 & 0.61 & 34.79 & 198.4 & 0.68 \\
\hline $18-\mathrm{CH}^{19-\mathrm{CH}_{2}}$ & 46.36 & 193.1 & 0.5 & 45.26 & 194.48 & 0.46 \\
\hline $20-\mathrm{CH}_{2}$ & 111.29 & 61.55 & 0.37 & 113.51 & 64.64 & 0.57 \\
\hline $21-\mathrm{CH}_{2}$ & 143.19 & -25.77 & 0.98 & 140.14 & -29.37 & 0.79 \\
\hline $22-\mathrm{CH}_{2}$ & 140.07 & -34.72 & 0.3 & 147.56 & -27.44 & 0.85 \\
\hline $23-\mathrm{CH}_{2}$ & 143.49 & 27.81 & 0.62 & 143.72 & 27.82 & 0.68 \\
\hline $24-\mathrm{CH}_{2}$ & 146.73 & -29.8 & 0.36 & 145.08 & -24.26 & 1 \\
\hline $\mathrm{N}-1$ & 155.01 & -63.66 & 0.38 & 157.18 & -68.72 & 0.33 \\
\hline $\mathrm{N}-2$ & 178.6 & -27.7 & 0.79 & 167.68 & 24.26 & 0.98 \\
\hline $\mathrm{N}-3$ & 47.5 & 125.65 & 0.32 & 47.6 & 124.22 & 0.35 \\
\hline & & & & & & \\
\hline
\end{tabular}

